\title{
The Role of Mediastinoscopy for Diagnosis of Isolated Mediastinal Lymphadenopathy
}

\author{
Zubin M. Nalladaru • Andre Wessels
}

Received: 20 July 2010 / Accepted: 5 January 2011 /Published online: 30 April 2011

(C) Association of Surgeons of India 2011

\begin{abstract}
To determine the role of mediastinoscopy (MDS) in the histological diagnosis of isolated mediastinal lymphadenopathy in a centre where more sophisticated investigations, like positron emitting tomography (PET) scan and endobronchial ultrasound guided trans-bronchial needle aspiration (EBUS-TBNA) is not available. This is a retrospective study. From January 2006 to December 2009, 31 patients with isolated mediastinal lymphadenopathy underwent MDS to establish a histological diagnosis. The mean age was 36.3 years. Among the patients, 19 were males and 12 were females. The most common symptom was dry cough in $18(58 \%)$ patients. The diagnostic yield was $96.8 \%$, with sarcoidosis being the commonest diagnosis. It was concluded that MDS was vital in establishing a histological diagnosis in patients with isolated mediastinal lymphadenopathy.
\end{abstract}

Keywords Mediastinoscopy - Lymphadenpathy ·

Sarcoidosis $\cdot$ Tuberculosis $\cdot$ Lymphoma

\section{Introduction}

Mediastinoscopy (MDS) is used for the diagnosis of thoracic disease and staging of malignancies since its introduction in 1959 by Carlens [1].

Non-invasive means, such as positron emitting tomography (PET) scans have recently raised doubts as to the importance of MDS in the diagnosis and staging of non-

Z. M. Nalladaru $(\triangle) \cdot$ A. Wessels

The City Hospital, Department of Cardiothoracic Surgery, Dubai Health Care City,

PO Box 505004, Dubai, United Arab Emirates

e-mail: zubin.nalladaru@thecityhospital.com small cell lung cancer with sensitivity and specificity almost approaching that of MDS [2]. However, since histological diagnosis is the issue, especially where a differentiation needs to be made between sarcoidosis, tuberculosis (TB) and lymphomas, MDS remains the diagnostic procedure of choice.

The aim of our study was to evaluate the role of MDS in cases with isolated mediastinal lymphadenopathy presenting as a diagnostic dilemma and to determine its safety and efficacy.

\section{Material and Methods}

All the patients were referred from the pulmonology or internal medicine service in our hospital and from clinicians in the community.

The institutional review and ethics board did not require individual patient consent as the study was retrospective.

All cases of isolated mediastinal lymphadenopathy undergoing MDS for diagnostic purposes between January 2006 and December 2009 were included in this study.

Cases with associated lung cancer and those with other parenchymal lung lesions were excluded from this study. In patients suspected to have a lymphoma, there were no subcutaneous peripheral lymph nodes accessible to biopsy under local anesthesia.

All patients had undergone extensive investigations including chest X-rays, computerised tomography (CT) scans, sputum cultures, serum angiotensin converting enzyme (ACE) test for sarcoidosis, and bronchoscopy with no diagnostic yield.

MDS was performed when the lymph node size was $>1 \mathrm{~cm}$ in its short axis on CT scan. MDS was carried out under general anesthesia with single lumen tracheal intubation, 
according to standard described techniques [3]. A fiberoptic bronchoscopy was routinely performed, if not done earlier. The intended lymph node for biopsy was always aspirated to prevent a vascular structure from being inadvertently biopsied. Multiple punch biopsies from various lymph node stations were obtained. The specimens were sent for histopathology, gram and acid fast bacilli (AFB) stain, and routine AFB cultures. We did not perform extended cervical MDS, described by Ginsberg and colleagues [4] on any of our patients. All patients were discharged 4-6 $\mathrm{h}$ after the procedure.

Data were then entered into a previously established database. This database was used to delineate the indications, result, and complications of all MDS performed by members of our service during the study period.

\section{Results}

A total of 31 patients underwent MDS for isolated mediastinal lymphadenopathy. Of these, 19 were male and 12 female $(\mathrm{M}: \mathrm{F}=1.6: 1)$. The age range was $18-52$ years with a mean of 36.3 years. The United Arab Emirates (UAE) population is a multicultural mix with a predominance of South Asians.

Among the patients, 25 were symptomatic on admission and 6 were asymptomatic. The commonest symptom was cough $(n=18 ; 58 \%)$ followed by fever $(n=8 ; 25.8 \%)$ and weight loss $(n=3 ; 9.6 \%)$.

Of the 31 patients undergoing MDS, biopsy was possible in 30 patients. The patient in whom biopsy was not possible turned out to have a vascular mass extending from the anterior mediastinum and enveloping the origin of the left common carotid and left subclavian arteries. Biopsy was deferred in this case as all attempts at needle aspiration resulted in drawing blood. This patient was subsequently explored through a mid sternotomy and underwent de-bulking of the mass. The entire mass could not be removed due to infiltration in the vascular structures. Histopathology revealed an invasive thymoma and the patient was referred by our oncology department for radiation therapy.

One patient had a pre-operative left laryngeal nerve palsy. The biopsy revealed invasive type of non-Hodgkins lymphoma. There were no operative deaths $(n=0 ; 0 \%)$. However, three patients developed minor post operative complications $(n=3 / 31 ; 9.6 \%)$. This included two cases of temporary hoarseness of voice, presumably due to traction injury to the left recurrent laryngeal nerve during biopsy of the left paratracheal lymph nodes. Both these patients recovered full voice function within a few weeks following the procedure. There was one case of late wound infection in a patient who biopsied positive for TB. This patient developed an abscess at the incision site 5 months following the MDS. The abscess was incised and drained and was allowed to heal with secondary intention. The wound healed without sinus formation. There were no major complications, such as catastrophic bleeding, pneumothorax, injury to trachea or oesophagus, which was reported in some larger series [5].

A total of 35 lymph node stations were sampled in 31 patients. The mean number of lymph node stations sampled per patient was 1.2 .

Of the samples, $57.1 \%$ were taken from the right paratracheal group, $17.2 \%$ from left para tracheal group, $17.25 \%$ from the pre-tracheal group, and $8.5 \%$ from the sub-carinal group. The histopathology results included sarcoidosis in $18(58 \%)$ patients, lymphoma in $6(19 \%)$, $\mathrm{TB}$ in $4(13 \%)$, reactive hyperplasia in $1(3.2 \%)$, and normal histology in $1(3.2 \%)$ patients. In one case, a biopsy could not be taken due to the persistent aspiration of blood from the intended biopsy site.

MDS resulted in a definitive diagnosis in 30 out of 31 (96.8\%) patients with isolated mediastinal lymphadenopathy.

\section{Discussion}

In this series, a total of 31 patients underwent MDS to establish a histological diagnosis of mediastinal lymphadenopathy in the absence of any identifiable parenchymal or endobronchial lesion. Of these patients, a majority $(n=24$, $77.4 \%$ ) had a benign process on histopathology. This is consistent with data found in other reports [6]. In both benign and malignant conditions, a precise histological diagnosis is required to decide the further course of management and thus a strong case can be made for MDS in most cases of isolated mediastinal lymphadenopathy.

The benefits of an accurate diagnosis obtained by MDS needs to be balanced against the risk of the procedure. Although MDS is a minimally invasive procedure, it should not be taken lightly as catastrophic complications have been reported in literature. Operative bleeding, post operative pneumothorax, wound infection, and recurrent laryngeal nerve palsy are infrequent complications. Rare instances of tracheal and oesophageal injuries have been reported [5]. Our mortality and morbidity rates compared with those published in literature.

In this series, MDS confirmed a definitive histological diagnosis in the majority of patients. This differentiated patients with sarcoidosis from those with TB and lymphomas. A surprise finding was the high prevalence of sarcoidosis in a predominantly South Asian population, in whom TB would be the most probable diagnosis. This resulted in the prevention of initiating empirical anti-TB pharmacotherapy. 
Chamberlain procedure or anterior MDS [7] gives direct access to aorto-pulmonary window lymph nodes, which are inaccessible by MDS and thus used to be an important adjunctive procedure. However, with the advent of newer sampling methods, such as video-assisted thoracoscopic biopsy and CT-guided trans-thoracic biopsy, it is now rarely performed.

Large and bulky mediastinal nodes are easily accessible to CT-guided trans-thoracic needle biopsy. This technique gives adequate yield and sufficient material for sophisticated pathological tests including immunohistochemistry and reverse transcription polymerase chain reaction (RT-PCR) and should be the first diagnostic option. Enlarged lymphnodes at the hilum, which are not accessible to MDS, can also be approached by CT guided biopsy. The ability to carry out CT-guided trans-thoracic needle aspiration for the diagnosis and staging of cancer in the mediastinum has generally been reported to be high. In a study by Bilaceroglu et al. [8], they reported a sensitivity of $82 \%$, although approximately $12 \%$ of patients required the placement of a catheter for the evacuation of a pneumothorax.

Video-assisted thoracoscopic surgery, also known as VATS, can be used to access mediastinal nodes. This is done under general anesthesia and in general is limited to an assessment of only one side of the mediastinum. Access to the right sided nodes is straightforward, but access to the left para-tracheal nodes is more difficult [9]. Other methods for obtaining histology specimens of enlarged mediastinal lymph nodes include CT guided trans-bronchial needle aspiration (TBNA) and endobronchial ultrasound guided trans-bronchial needle aspiration (EBUS-TBNA). These methods are currently not available in our institute and need to be explored as they are less invasive than MDS and do not require a general anaesthetic.

Combined PET-CT is another very useful non-invasive tool in our armamentarium to differentiate malignancies from non-malignant mediastinal lymphadenopathy. It is especially useful in the staging and follow-up for lung cancer. However, with the currently available technology, it can diagnose non-malignant lesions but cannot differentiate between them [10].

In conclusion, MDS and biopsy continues to be an important tool for the diagnosis of isolated mediastinal lymphadenopathy. It is effective and very safe in experienced hands.

\section{References}

1. Carlens E (1959) Mediastinoscopy: a method for inspection and tissue biopsy in the superior mediastinum. Dis Chest 36:343-349

2. Lee BE, Redwine J, Foster C, Abella E, Lown T, Lau D, Follette D (2008) Mediastinoscopy might not be necessary in patients with non-small cell lung cancer with mediastinal lymph nodes having a maximum standardized uptake value of less than 5.3. J Thorac Cardiovasc Surg 135:615-619

3. Kirschner PA (1996) Cervical mediastinoscopy. Chest Surg Clin N Am 6(1):1-20

4. Ginsberg RJ (1996) Extended cervical mediastinoscopy. Chest Surg Clin N Am 6:20-30

5. Hammoud ZT, Anderson RC, Myers BF, Guthrie TJ, Roper CL, Cooper JD, Patterson GA (1999) The current role of mediastinoscopy in the evaluation of thoracic disease. J Thorac Cardiovasc Surg 118:894-899

6. McManus TE, Haydock DA, Alison PM, Kolbe J (2008) Isolated mediastinal adenopathy: the case for mediastinoscopy. Ulster Med J 77(2):97-101

7. Olak J (1996) Parasternal mediastinotomy (Chamberlain procedure). Chest Surg Clin N Am 6(1):31-40

8. Bilaceroglu S, Chhajed P, Mavis A, Aydogdu Z, Cagirici U (2007) Computed tomography-guided transthoracic needle aspiration in diagnosing and staging hilar and mediastinal masses of lung cancer after negative bronchoscopy. J Bronchol 4:149-155

9. Massone PP, Lequaglie C, Magnani B, Ferro F, Cataldo I (2003) The real impact and usefulness of video-assisted thoracoscopic surgery in the diagnosis and therapy of clinical lymphadenopathies of the mediastinum. Ann Surg Oncol 10:1197-1202

10. Crina FM Mediastinoscopy more reliable than PET for characterizing granulomatous inflammation in lymph nodes. Poster \# 29 American society for clinical pathology, annual meeting. Presented October 17, 2008. 